\title{
TTR
}

Traduction, terminologie, re?daction

\section{Henri Van Hoof. Dictionnaire universel des traducteurs. Genève, Éditions Slatkine, 1993, 414 p.}

\section{Jean Delisle}

Volume 6, numéro 2, 2e semestre 1993

Traduction, mixité, politique

URI : https://id.erudit.org/iderudit/037155ar

DOI : https://doi.org/10.7202/037155ar

Aller au sommaire du numéro

\section{Éditeur(s)}

Association canadienne de traductologie

ISSN

0835-8443 (imprimé)

1708-2188 (numérique)

Découvrir la revue

Citer ce compte rendu

Delisle, J. (1993). Compte rendu de [Henri Van Hoof. Dictionnaire universel des traducteurs. Genève, Éditions Slatkine, 1993, 414 p.] TTR, 6(2), 158-163.

https://doi.org/10.7202/037155ar d'utilisation que vous pouvez consulter en ligne.

https://apropos.erudit.org/fr/usagers/politique-dutilisation/ 


\section{Henri Van Hoof. Dictionnaire universel des traducteurs. Genève, Éditions Slatkine, 1993, 414 p.}

Vous désirez connaître les principales œuvres traduites par Maurice Rambaud. Vous ne vous souvenez plus si Prosper Mérimée a traduit Gogol ou Dostoïevski. Vous voulez vérifier la date de naissance du lexicographe-traducteur tchèque Josef Jungmann. Vous lisez sur la page titre $d^{\prime}$ 'une cuvre traduite le nom d'une traductrice (inscrit en petits caractères, évidemment) et, par curiosité, vous désirez en savoir plus long à son sujet. Vous vous interrogez sur le siècle où a vécu le traducteur Bonaventura Tecchi. Vous hésitez sur la nationalité d'Henri Amiel: Est-il Français, Belge ou Suisse? Vous vous demandez si le traducteur suédois Carl Gustav Bjurström a obtenu un prix de traduction et, si oui, lequel. Tous ces renseignements, vous les trouverez dans le Dictionnaire universel des traducteurs, neuvième ouvrage publié par Henri van Hoof. Qui est Henri van Hoof? Ceux qui ne le connaîtraient pas encore pourront ouvrir son dictionnaire et lire la notice suivante à la page 383:

\section{VAN HOOF Henri (1923- )}

1. Professeur et traducteur belge d'expression française, né à Watermael-Boitsfort/Bruxelles. A enseigné la théorie et la pratique de la traduction à l'Institut supérieur pour Traducteurs et Interprètes M. Haps (Bruxelles (1956-82)). Cofondateur et ancien président de la Chambre belge des Traducteurs et Interprètes. Cofondateur et rédacteur en chef de la revue $L \boldsymbol{E}$ Linguiste (1955-77). Traducteur juré près la Cour d'Appel de Bruxelles. Auteur d'une Terminologie économique anglais-français. 2. A traduit du néerlandais: L'Expansion de la gravure flamande aux Indes, en Chine et au Japon auX XVF et XVII siecles (1949) de R.P. Jennes; de l'anglais: Thomas Hardy (1949) de H.W. Nevinson; en néerlandais: des négro spirituels et des chansons américaines dans Amerika Nu (1959) de Jan Boon (1898-1960); en anglais: Christophe Colomb (1954), pièce de Charles Bertin (1919), La grande tentation de saint Antoine (1957), pièce radiophonique de Michel Ghelderode (1898-1962), etc.

Auteur de nombreux articles sur la traduction et de plusieurs ouvrages comme Théorie et pratique de l'interprétation (1963), Bibliographie internationale de la traduction (1973), Petite histoire de la traduction en Occident (1986), Précis pratique de traduction médicale (1986), Traduire l'anglais (1989), Histoire de la traduction en Occident (éd. revue et augmentée, 1991), etc. 
On remarque à la lecture de cette notice que les deux principaux champs d'intérêt de l'auteur sont l'histoire et la pédagogic de la traduction. Au cours de sa carrière déjà longue, $M$. van Hoof a voulu doter la profession d'instruments de recherche et de formation. En publiant ce dictionnaire universel, il souhaite en outre apporter sa contribution au projet d'histoire générale de la traduction de la Fédération internationale des traducteurs (FIT) en recensant les noms de centaines $d$ 'hommes et de femmes qui ont exercé dans le passé ou exercent actuellement le métier de traducteur ou de traductrice. Il est d'ailleurs membre du Comité pour l'histoire de la traduction (FIT).

Sur une période de trente ans, Henri van Hoof a accumulé patiemment des milliers de fiches. Tenter de relever tous les noms de ceux et celles qui ont fait ou font de la traduction depuis l'invention de l'écriture aurait constitué un projet utopique, été d'une ambition démesurée. Des choix s'imposaient donc. Comment l'auteur a-t-il procédé? Il nous le dit dans son Avant-propos: "C'est l'accès aux sources qui a conditionné la sélection finale» (p. vii). Et ces sources quelles sont-elles? Ce sont les catalogues de bibliothèques, encyclopédies, histoires de la littérature, ouvrages spécialisés, dictionnaires d'auteurs, revues de traduction, le tout en plusieurs langues. Il a aussi interrogé des traducteurs, consulté des associations professionnelles, des maisons d'édition, des fondations de prix. Modeste, $H$. van Hoof ne se fait pas d'illusion sur l'exhaustivité de son travail: «ll faut bien admettre en toute humilité que le résultat paraîtra toujours disproportionné par rapport à l'ampleur de la tâche. $C^{\prime}$ est dire si nous savons d'avance combien cet inventaire est incomplet" (p. vii).

Les notices du dictionnaire épousent toutes le même schéma: un premier bloc d'informations donne des éléments biographiques et précise les facteurs éclairant l'activité traduisante. Suit une liste des titres traduits et, le cas échéant, des prix obtenus par les auteurs des traductions. Examinons maintenant de plus près le contenu de l'ouvrage.

Comme il fallait $s^{\prime} y$ attendre, on $y$ trouve les incontournables de la traduction, les Cicéron, Amyot, Dolet, Chaucer, Florio, Wyclif, ibn Ishâq, Caxton, North, Galland, etc., de même que les grands auteurs qui n'ont pas dédaigné s'adonner à cette activité, 
les Chateaubriand, Baudelaire, Dostoïevski, Yourcenar, Yeats, Gide, Dryden, etc. Il y a aussi des saints (Jérôme, Cyrille), des papes (Paul VI), des princes et des souverains (Catherine II de Russie, Élisabeth $1^{\text {ère, }}$ Alphonse $X$, roi de Castille, etc.). Si l'on en juge par le contenu de la notice consacrée à Alphonse $X$, celui-ci n'a pas fait de traduction, mais a plutôt été un promoteur de la traduction. Les mécènes et les protecteurs des traducteurs, si utiles soient-ils, sont-ils vraiment à leur place dans un dictionnaire de traducteurs? Si oui, il faut alors inclure aussi bon nombre de rois de France (Philippe IV le Bel, Jean II le Bon, Charles V, etc.) et plusieurs rois d'Angleterre, d'Espagne et de nombreux autres pays. Cela risque de nous entraîner loin et de reléguer encore une fois les traducteurs dans l'ombre.

Il en est de même pour les prix de traduction, dont certains font l'objet d'une entrée. Exemple: Prix de traduction HalpérienKaminsky, John Glassco, Fiumi-Leroux, FIT-Nathorst (sic) [le nom officiel de ce prix est Carl-Bertil Nathhorst], etc. Quels critères ont présidé à l'inclusion de certains prix et à l'exclusion d'autres distinctions? Si l'on examine le traitement réservé aux seuls prix attribués par la FIT, aucune mention n'est faite, sauf erreur, des prix Astrid Lindgren, Karel Capek et de la médaille Pierre-François Caillé. Pourtant les deux derniers traducteurs, l'un Tchèque, l'autre Français, ont droit à une notice. La meilleure manière de traiter les prix de traduction aurait été, nous semble-t-il, de les regrouper dans un Appendice en fin de volume. Cela aurait exigé cependant de procéder de façon systématique par enquete auprès de toutes les sociétés nationales de traduction afin d'établir la liste la plus complète possible des grands prix nationaux et internationaux. L'auteur aurait ainsi atténué la part d'arbitraire, de "hasard», qui a prévalu à cet égard.

À côté des "vedettes" de la traduction et des Grands de ce monde défile la longue cohorte $d^{\prime}$ "illustres inconnus" sur lesquels le dictionnaire jette un peu de lumière. On peut même dire que c'est ici que l'ouvrage se révèle le plus utile car, si tout bon dictionnaire des noms propres nous renseigne sur les Dolet, Amyot, Caxton et autres grandes figures de la profession, ils restent lamentablement muets lorsqu'il s'agit des traducteurs peu connus. Certaines notices sont plus étoffées que d'autres. Leur longueur n'est pas fonction de l'importance relative du personnage, mais tributaire encore une fois 
des sources documentaires consultées. L'article consacré à Rudolf Bach (1901-1957) est assez représentatif d'une «notice pauvre", dont le nombre, il faut le dire, est limité: «1. Dramaturge, essayiste et poète allemand, né et mort à Munich. 2. Également traducteur.» En revanche, les notices de Jean Lascaris, Vassili Joukovski, Philippe Jaccottet, Claude Couffon couvrent une bonne demi-colonne de ce dictionnaire grand format (il fait $22 \mathrm{~cm}$ sur $30 \mathrm{~cm}$ ), celle de Françoise Campo-Timal, deux tiers de colonne et celle de Carl Gustav Bjurström plus d'une colonne. La majorité des notices comptent de cinq à dix lignes. En outre, sans avoir procédé à un décompte statistique rigoureux, il nous a semblé que le dictionnaire renferme une forte proportion de traducteurs et de traductrices d'expression française. Viennent ensuite les Anglais, les Américains, les Allemands, les Néerlandais, les Italiens. Le hasard des sources?

À propos d'Italiens, l'un d'eux a droit à deux notices distinctes. Il s'agit de l'auteur-traducteur Gian Francesco Poggio Bracciolini (1389-1459), qui a contribué à répandre l'écriture humanistique [abandon de l'écriture gothique au profit de la caroline] et qu'on appelle communément en français Le Pogge. Dans le dictionnaire, il figure sous le nom de «Poggio BRACCIOLINI" et sous celui de "POGGIO BRACCIOLINI, Gian Francesco". Heureusement, les renseignements fournis à son sujet dans les deux notices ne se contredisent pas, la première offrant plus de détails que la seconde. Par ailleurs, une légère imprécision s'est glissée dans l'article de saint Cyrille. Cet «apôtre des Slaves» n'a pas inventé à strictement parler l'alphabet cyrillique, mais l'alphabet glagolitique, dont dérive le cyrillique. C'est d'ailleurs ce que $M$. van Hoof écrivit lui-même dans un article qu'il fit paraître dans Babel (XXXVI-1, pp. 39-40), "Traduction biblique et genèse linguistique", preuve qu'il faut voir dans cette imprécision une simple faute d'inattention de sa part.

Devant une entreprise de compilation d'une telle envergure, signaler des omissions ou des oublis relève quasiment de la mesquineric, tant la collecte des données rassemblées par l'auteur a exigé de lui effort et persévérance et tant la tâche à accomplir était titanesque. Toutefois, M. van Hoof invite lui-même le lecteur à lui «signaler les omissions importantes", à lui fournir "des précisions complémentaires" (p. vii). Nous nous contenterons de signaler quelques noms. Ainsi, Cyrille et Wulfila sont dans le dictionnaire, mais par l'Arménien Mesrop Machtots (l'inventeur des alphabets 
arménien et georgien) ni le frère de Cyrille, Méthode, qui traduisit tout autant que son cadet. De même, chez les contemporains français, François Maspero et la traductrice de Kafka, Marthe Robert, $\mathrm{n}^{\prime} \mathrm{y}$ sont pas, alors qu'Alexandre Vialatte, Pierre Leyris, Edmond Cary et Pierre-François Caillé y figurent. Nous signalons ces quatre omissions pour montrer que le plus difficile dans un travail de ce genre est de définir clairement au point de départ un mode de sélection significatif en fonction de critères opérationnels. L'aspect méthodologique est très important. La cohérence de l'ouvrage en dépend. Puisque les auteurs jouissent en général d'une rénommée plus grande que leur(s) traducteur(s), il aurait été utile, à notre avis, d'ajouter un index des noms d'auteurs avec en regard celui de leur(s) traducteur(s). Ainsi, pour connaître le principal traducteur de l'écrivain autrichien Peter Handke, l'index nous aurait renvoyés à Georges-Arthur Goldschmidt.

Enfin, signalons que le Dictionnaire universel des traducteurs réserve une place à certains traductologues contemporains, dont $\mathrm{E}$. Nida, A. Berman, G. Mounin, J. Darbelnet, J.-P. Vinay, T. Savory, W. Wilss, A. Lefevere, E. Etkind, J.C. Catford. Quant aux Canadiens, ils y sont assez bien représentés. Des articles sont consacrés à $P$. Daviault, J. Glassco, S. Fischman, I. de Buisseret, P. Claxton, G. Panneton, J. Delisle, G. Dagenais, P. Horguelin, R. Dubuc, M. Laurence, J. Darbelnet et J.-P. Vinay.

En somme, bien que l'on puisse chicaner amicalement l'auteur sur sa méthode de compilation et sur certaines erreurs de détail, il demeure qu'il a produit une ceuvre utile. Son dictionnaire est bien sûr perfectible, mais quel travail de pionnier ne l'est pas? L'auteur considère d'ailleurs lui-même son inventaire comme un "point de départ» et souhaite recevoir l'aide de personnes désireuses de participer activement "à cet hommage [rendu] au traducteur de tous les temps" (p. vii). C'est un travail colossal et ingrat qu'a entrepris $M$. van Hoof, il y a trente ans, sans équipe de recherche et sans subvention. Il faut le féliciter d'avoir gardé le feu sacré pendant toutes ces années et $\mathrm{d}^{\prime}$ avoir consigné sur ses milliers de fiches des renseignements souvent difficiles à trouver concernant les travailleurs de l'ombre que sont les traducteurs. Ce dictionnaire vient s'ajouter utilement à celui de Paul Chavy, Traducteurs d'autrefois. Moyen Àge et Renaissance. Dictionnaire des traducteurs et de la littérature traduite en ancien et moyen français (842-1600) publié aussi aux éditions 
Slatkine, en 1988 (voir TTR, II-1, 1989, pp. 163-169). Petit à petit s'enrichit la bibliothèque de l'histoire de la traduction.

Jean Delisle Université d'Ottawa 